\title{
A combined anterior and posterior approach for thoracolumbar spinal tuberculosis
}

\author{
Bo Wang ${ }^{1,4 *}$, Dawei Tian ${ }^{2}$, Juan Zhou ${ }^{3}$ and Kunzheng Wang ${ }^{4 *}$ \\ ${ }^{1}$ Department of Spine Section, University of Chinese Academy of Sciences-Shenzhen Hospital, Shenzhen 518007, Guangdong, China \\ ${ }^{2}$ Department of Orthopedics, Hubei University of Medicine Affiliated Dongfeng Hospital, Shiyan 442008, Hubei, China \\ ${ }^{3}$ Department of Medicine Education, University of Chinese Academy of Sciences - Shenzhen Hospital, Shenzhen, Guangdong 518007, China \\ ${ }^{4}$ Department of Medicine, Joint Center, Xi'an Jiaotong University, Xi'an, Shaanxi 710061, China
}

\begin{abstract}
Spinal tuberculosis is a common extrapulmonary tuberculosis, causing abscess formation or bone destruction. With a climbing multidrug resistance of Mycobacterium tuberculosis, it remains highly prevalent. As spinal cord compression causes high disability rate, surgical intervention is a much-needed option. However, an optional surgical approach is still highly desirable. We have reported a one-stage surgical protocol for twenty-six cases with thoracic and lumbar tuberculosis. Eight of them had thoracic tuberculosis and eighteen were thoracolumbar tuberculosis. In addition to antituberculosis therapy, we approached with posterior pedicle screws for internal fixation, interlaminar bone graft and anterior debridement combined with autogenous bone grafting. After a longitudinal follow-up as long as 36 months, symptoms of lumbago and back pain were relieved with functional nerve recovery. This study is an update for the patients with thoracolumbar tuberculosis who were not well treated with non-operative treatment, optimal clinical outcome can be achieved by following a one-stage operation protocol.
\end{abstract}

\begin{abstract}
Abbreviations: CRP: C-Reactive Protein; CT: Computed Tomography; EM: Ethambutol; ESR: Erythrocyte Sedimentation Rate; INH: Isoniazid; MRI: Magnetic Resonance Imaging, PZA: Pyrazinamide; RFP: Rifampin; SM: Streptomycin; TB: Tuberculosis; VAS: Visual Ana$\log$ Scale.
\end{abstract}

\section{Introduction}

Tuberculosis of spine (TB) is a common form of bone and joint tuberculosis. In the past decades, it has become more prevalent due to climbing multidrug resistance to Mycobacterium tuberculosis. Spinal tuberculosis is the most common transmission of extrapulmonary tuberculosis, where patients usually appear to have bone destruction and abscess formation that may compress spinal cord, causing movement disability or paralysis [1]. Treatment for tuberculosis with drugs and surgical intervention is a much-needed option, but there is still plenty room to improve surgery outcomes. In general, the goal of a surgery is to achieve best bone grafting, so that spinal deformity may be prevented, and the biomechanical stability of spines may be restored [2]. In this study, we performed a one-stage surgery for thoracic and lumbar tuberculosis to achieve posterior correction through a combination of internal fixation, anterior debridement and autogenous bone grafting [3].

\section{Materials and methods}

\section{Participants and baseline characteristics}

Twenty-six cases of thoracic and lumbar spinal tuberculosis were recruited from September 2010 through March 2016 (Male, 17; Female, 9; aged 17- 66 years). Patients have a sickness history of six to thirtysix months. Active tuberculosis diagnosis was initially performed based on the following criteria: 1) apparent clinical symptoms (weight loss, low fever, night-sweats and fatigue); 2) laboratory findings (high erythrocyte sedimentation rate (ESR) and C-reactive protein (CRP); 3) radiographic imaging indicating abnormal intensity of involved vertebrae, paravertebral or psoas abscesses $[4,5]$. The candidates identified for surgery were based on the following indications: 1) progressive neurological deficits; 2) persistent pain due to spinal instability; 3) severe kyphosis or progressive kyphosis; 4) poor beneficial outcomes following previous therapeutic drugs. Patients with active pulmonary tuberculosis were excluded.

Among the patients for surgeries, nine had spinal compression according to Frankel classification; three belonged to $\mathrm{C}$ grade, and six were determined as D class. For those of impaired T3-S1 segments, five had three segments, and sixteen had two sections. Only a few patients had chronic fever, night sweating, emaciation or other systemic symptoms. All patients had initial imaging by X-Ray, computed tomography (CT) or magnetic resonance imaging (MRI) (Figures 1-3). Eight of them involved thoracic vertebrae; six had thoracolumbar tuberculosis; twelve had lumbar tuberculosis below L2. For one case, three vertebral bodies were affected. Nineteen of them had a tuberculosis history; six had tuberculous pleurisy, and one had renal tuberculosis. The preoperative pain was 6.8 , and an average spinal kyphosis angle (Cobb angle) was 16.5 degrees [6].

${ }^{\star}$ Correspondence to: Kunzheng Wang, Department of Medicine, Joint center, Xi'an Jiaotong University, Xi'an, Shaanxi 710061, E-Mail:wangkz088@yeah.net

Bo Wang, Department of Spine Section, University of Chinese Academy of Sciences Shenzhen Hospital, Shenzhen 518003, Guangdong, China, E-mail: bo_wang-sz@outlook.com,same7213@163.com

Key words: thoracic vertebrae, lumbar vertebrae, spinal tuberculosis, surgical procedure

Received: November 13, 2020; Accepted: November 23, 2020; Published: November 30, 2020 


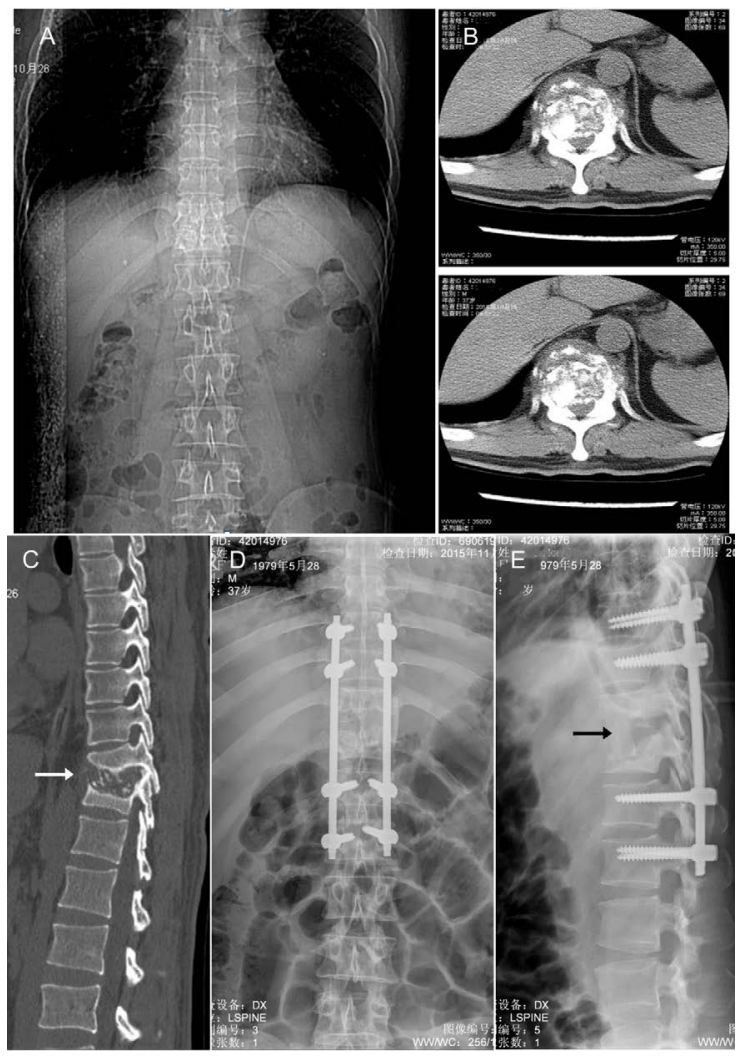

Figure 1. X-ray images of severe vertebral destruction and abscess formation. (A). A representative of preoperative X-ray photograph indicates the location of damaged thoracic spine; (B). A close-up view of destruction in a preoperative CT image; (C). A lateral view of the preoperative damage in CT (arrow); (D, E) X-ray images show in positive and lateral position of the pedicle screws for fixed spine after operation. All images were taken from the same patient.
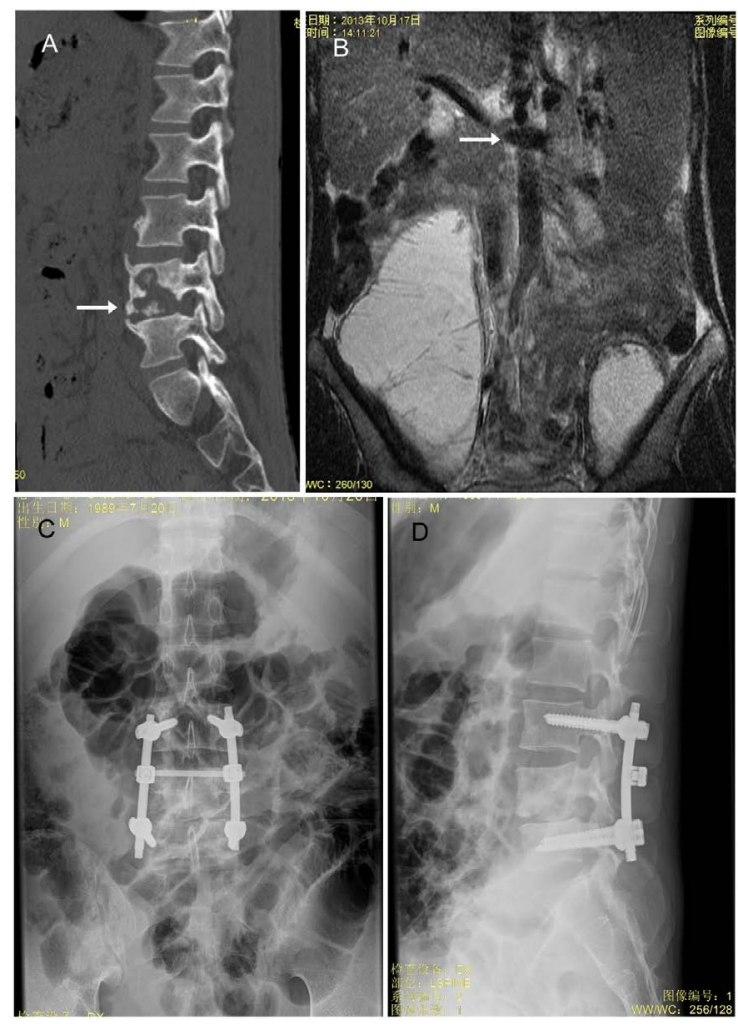

Figure 2. CT images of thoracic spine before and after surgery. (A). A representative of destructed lower thoracic spine (arrow) is indicated in a preoperative CT image; (B). The location of damaged spine is shown in a preoperative MRI photo; (C, D) X-ray images show back and lateral positions of pedicle screws after operation. 


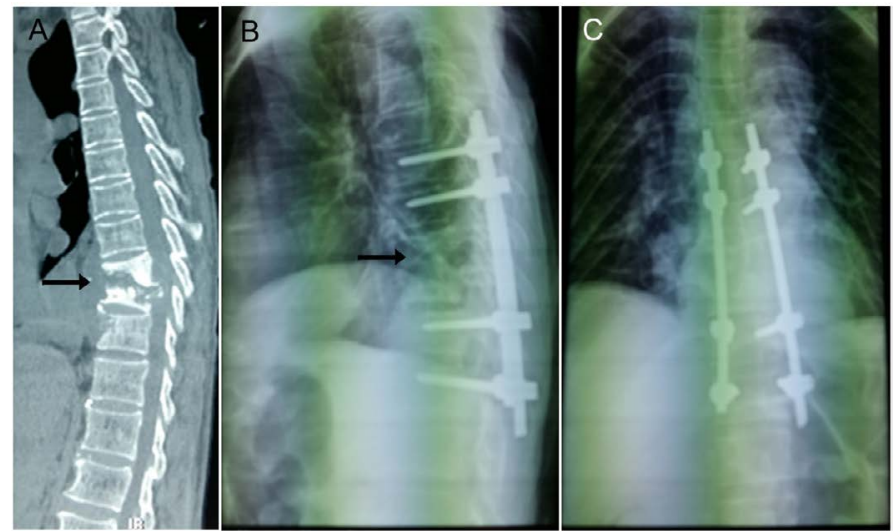

Figure 3. (A). A representative image shows destructed upper thoracic spine (arrow) in a preoperative $\mathrm{CT} ;(\mathrm{B}, \mathrm{C})$. X-ray indicates that lateral and back position of pedicle screws and the fixed spine (arrow) after operation.

\section{Treatment methods}

In preparation of surgery, a preoperative care was serviced for all incoming patients, that anti-tuberculosis drugs were treated for more than three weeks (outpatient). Malnutrition and hypoproteinemia were improved as well if necessary. The anti-tuberculosis program had adopted a quadruple chemotherapy including Isoniazid (INH), Rifampin (RFP), Ethambutol (EM), Pyrazinamide (PZA) or Streptomycin (SM), and the ESR was reduced significantly after treatments (Table 1). A re-evaluation confirmed that there was no active tuberculosis in spinal column.

The surgery operation commenced under a general anesthesia by following a procedure of tracheal intubation. First, a patient was positioned in supine position, and a posterior spinal median incision was made. The segmental vertebral body or adjacent vertebral bodies were fixed with two to four pedicle screws. For those subjects with intervertebral space involvement and lower vertebral destruction, two pairs of screws were used to fix adjacent vertebral bodies with the affected intervertebral space (Figures 1-3). When approaching to the focus, every possible effort was made to avoid passing across normal vertebral bodies. Orthodontic kyphotic deformity, segmental lamina and small joints of lesion were treated with artificial bone graft. Next, the patient was positioned in lateral position. This followed with a removal of the anterior debridement, caseous necrosis tissue, dead bone and bone graft fusion. Two patients had bilateral incisions. For thoracolumbar tuberculosis, a thoracic approach was done to perform a renal incision through the chest, extraperitoneal or transcostal transverse processes. Extraperitoneal and extraperitoneal approaches were also adopted for middle and lower lumbar tuberculosis. For lumbosacral tuberculosis, an abdominal median extraperitoneal approach was followed. With a focus being fully exposed, the purulent, dead bone, necrotic tissue and intervertebral disc were completely removed with a bone knife and curette. The compression of the spinal cord was significantly released upon the procedure being taken. In the spinal canal, repeated local irrigation was minimized. Further, a proper amount of autogenous iliac bone or rib was cut to correct the bone defect and a structural bone graft was made. For this procedure, a tight inlay pressure was carefully monitored. The focus and its surrounding were placed gelatin sponge wrapped with streptomycin $(1 \mathrm{~g})$ and isoniazid $(3 \mathrm{mg})$. Lastly, one phase suture incision was finalized without a drainage tube being placed. These procedures normally took 160 - $330 \mathrm{~min}$, typically costing of intraoperative bleeding of $300-1200 \mathrm{ml}$ [7].
After operation being taken for four weeks, limbs and back muscles of the patients were able to exercise such as fixed weight standing training [8]. During the period of 12-18 months of routine antituberculosis treatment, ESR, Cobb angles, liver and kidney function were monitored regularly, and X ray or CT, MRI imaging was followed up [9]. The ESR and cobb values were measured two weeks after surgery and exported to Matlab (Mathworks, MA) for data analysis. The pre-surgery or post-surgery values were fit with a Kernel function to present a trend of distribution; the original values were used for boxplot and median calculation and tested by performing unpaired t-test (Tables 1 and 2, Figure 4).

\section{Results}

The postoperative follow-up time was twelve to thirty-six months $(1,3,6,12,18,24,36$ mos) by lab tests, physical examination, and radiography [10]. Most of the patients being treated with one-stage combined approach for internal fixation had successful focus clearance and bone graft fusion (Figures 1-3). One patient formed sinuses but healed eventually; two cases had delayed healing. The average posterior Cobb angle was -3 (22 degrees corrected compared with the preoperative) (Table 2 and Figure 4B); the symptoms of low back pain was improved significantly with an average of VAS score of two. The improvement of nerve function was evaluated by Frankel classification: 1 case was grade D; the rest were grade $\mathrm{E}$. A follow-up examination of ESR and CRP were normal (Table 1 and Figure 4A) [11].
A

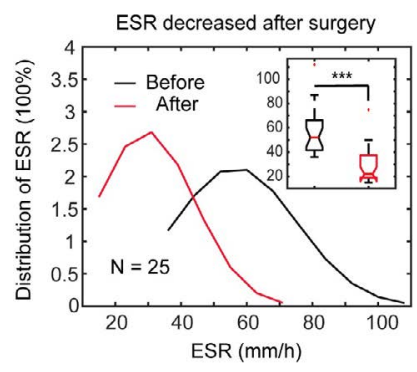

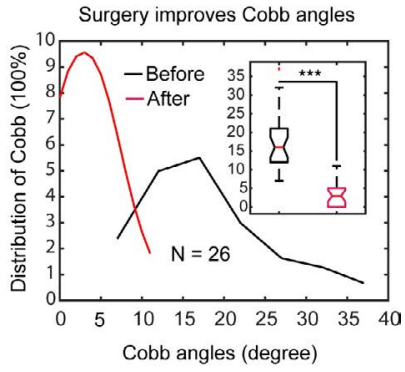

Figure 4. Preoperative ESR evaluation and Cobb angles correction following surgery. A ESR value distribution before and after quadruple chemotherapy. Inset. A boxplot of the ESRs. Asterisks indicate the ESR decreased significantly after treatment (t-test: $\mathrm{P}<0.001$ ). B. $\mathrm{Cobb}$ angles distribution before and after surgery. Inset, a boxplot of the Cobb angles. Asterisks indicate that the Cobb angles were corrected significantly by operation (t-test: $\mathrm{P}<0.001$ ).

Table 1. ESR of pre-and post-operation, ESR $(\mathrm{mm} / \mathrm{h})$

\begin{tabular}{|l|l|l|l|l|l|l|}
\hline & & $<\mathbf{4 0}$ & $\mathbf{4 0}-\mathbf{6 0}$ & $\mathbf{6 0 - 8 0}$ & $\mathbf{8 0}-\mathbf{1 0 0}$ & $>\mathbf{1 0 0}$ \\
\hline Patients & Preoperative & 6 & 11 & 7 & 1 & 1 \\
\hline & Postoperative (2 w) & 20 & 5 & 1 & 0 & 0 \\
\hline
\end{tabular}

Table 2. Cobb angles (Degree)

\begin{tabular}{|l|l|}
\hline Preoperative & $16.5 \pm 5.9$ \\
\hline Postoperative & $4.5 \pm 1.1$ \\
\hline Final follow-up & $6.4 \pm 0.9$ \\
\hline ASIA classification & 7 \\
\hline D & 17 \\
\hline E & \multicolumn{2}{|l|}{} \\
\hline VAS & $6.8 \pm 2.8$ \\
\hline Preoperative & $1.1 \pm 0.9$ \\
\hline Final follow-up & \\
\hline Complications & 2 \\
\hline Pain of graft harvesting site & \\
\hline
\end{tabular}

ASIA: American Spinal Injury Association; VAS: Visual Analog Scale 


\section{Discussion}

In as early as 1950s, removal of dead bone through anterior approach combined with bone grafting had been shown to improve recovery rate of spinal tuberculosis. More clinical practice had confirmed the importance of a removal of lesion and primary bone graft fusion [12]. In recent years, as a consequence of rising drug resistance to chemotherapy, physicians have concluded an consensus of the significance of active surgical TB treatment: 1) effective improvement of deformity and full decompression; 2) rapid fusion; 3) reduction of bone loss; 4) less bed time and rapid relief of pain; 5) less spinal tuberculosis recurrence rate [13]. With a combined operation and individualized chemotherapy, better biomechanical stability and reconstruction of physiological function can be accomplished [14]. It should be noted that basic principles of spine tuberculosis treatment should continue to be followed: general treatment (rest or nutrition) and regular chemotherapy [15]. As surgical indications for spinal tuberculosis include a destruction of dead bone in vertebral body, giant parvertebral abscess, compression of spinal cord and chronic sinus tract, a therapeutic goal has been to remove all the infectious tissues and dead bones, to relieve compression on spinal cord and to correct spinal kyphosis, thereby to restore spinal function [16].

There is an exception that a preoperative anti-tuberculosis treatment is urgently needed. Six patients in this study were treated with a prolonged period of anti-tuberculous therapy prior to surgery. The operation was taken when the ESR was $42-75 \mathrm{~mm} / \mathrm{h}$ (Figure $4 \mathrm{~A}$ and Table 1). The ESR value had once increased, accompanied with fever, but the symptoms gradually disappeared in three weeks. We did not hesitate to postpone the surgery by waiting for a normal ESR, because a delay may have us missed a favorable operation time, that may cause vertebral destruction, abscess enlargement, and even paralysis [17].

Anterior debridement, spinal cord decompression, and bone graft fusion are the most common surgical procedures for treatment of severe spinal tuberculosis, but sometimes kyphosis is aggravated due to bone graft bed absorption, false joint formation and bone block collapse. In a specific scenario, the bone graft is transferred to spinal cord, causing serious complications. This is because an internal fixation may fail to correct posterior protruding, and the posterior approach must be adopted. It should be noted that, as a lesion is not easy to be removed, spinal nerve root may be damaged to cause other nervous complications. Our approach can not only remove lesion completely, but also can correct a deformity well toward spine stabilization. Thus, it has advantages over the traditional procedures which need longer operation time and have greater risk to develop trauma. In a worse situation, a traditional protocol may complicate the disease condition, making it difficult to be operational. Therefore, the posterior internal fixation is more rational way that can achieve curative effect and reduce complications [16]. To conclude, one stage operation with posterior orthopedic fixation with anterior debridement and bone grafting can achieve therapeutic outcome for thoracolumbar spinal tuberculosis.

\section{Data sharing statement}

The datasets generated and/or analyzed during the current study that involve patient data are available upon request.

\section{Ethics and consent statement}

This retrospective study was approved by the Ethics Review Committee of the University of Chinese Academy of Sciences, Shenzhen Hospital (2010). Since this study was a retrospective comparative study, informed consent was waived by the ethics committee. All patient data were anonymously analyzed. All investigations were conducted in conformity with ethical principles of research.

\section{Disclosure}

The authors report no funding and no conflicts of interest in this work.

\section{References}

1. Yang H, Hou K, Zhang L, Zhang X, Wang Y, et al. (2016) Minimally invasive surgery through the interlaminar approach in the treatment of spinal tuberculosis: A retrospective study of 31 patients. J Clin Neurosci 32: 9-13.

2. Liu J-M, Zhou Y, Peng AF, Chen XY, Chen WZ, et al. (2017) One-stage posterior surgical management of lumbosacral spinal tuberculosis with nonstructural autograft. Clin Neurol Neurosurg 153: 67-72.

3. Yuvaraja M, Krishnan V, Amritanand R, Arockiaraj J, David KS (2016) Is Healing on MRI Mandatory for Discontinuing Antitubercular Therapy in Tuberculosis of Spine? The Spine Journal 16: S165-s166.

4. Lai Z, Shi S, Fei J, Han G, Hu S (2018) A comparative study to evaluate the feasibility of preoperative percutaneous catheter drainage for the treatment of lumbar spinal tuberculosis with psoas abscess. J Orthop Surg Res 13: 290.

5. Banga RK, Singh J, Dahuja A, Garg RS (2012) Spinal Tuberculosis - Directly Observed Treatment and Short Course or Daily Anti Tubercular Therapy -Are We Over Treating? Open Orthop J 12: 380-388.

6. Chen YH, Lin C-B, Harnod T, Wu W-T, Yu J-C, et al. (2013) Treatment modalities for tuberculosis of the spine: 22 years' experience in east Taiwan. Formosan J Surg 46: 189-194.

7. Page LL, Feydy A, Rillardon L, Dufour V, Le Hénanff A, et al. (2006) Spinal Tuberculosis: A Longitudinal Study with Clinical, Laboratory, and Imaging Outcomes. Semin Arthritis Rheum 36: 124-129. [Crossref]

8. Ekinci S, Akyildiz F, Ersen O (2015) A retrospective controlled study of three different operative approaches for the treatment of thoracic and lumbar spinal tuberculosis. Clin Neurol Neurosurg 136.

9. Cheung WY, Luk DK (2011) Tuberculosis of the spine. Orthopaedics and Trauma 25: 161-167.

10. Kaloostian EP, Gokaslan LZ (2013) Current Management of Spinal Tuberculosis: A Multimodal Approach. World Neurosurg 80: 64-65. [Crossref]

11. Almeida Á (2005) Tuberculosis of the spine and spinal cord. Euro J Radiol 55: 193-201.

12. Jain A, Dhammi I (2007) Tuberculosis of the spine: a review. Clin Orthopaedics Relat Res 460: 39-49.

13. Jutte P, Wuite S, The B, van-Altena,R, Veldhuizen A (2007) Prediction of deformity in spinal tuberculosis. Clin Orthop Relat Res 455: 196-201. [Crossref]

14. Zhao J, Lian XF, Hou TS, Ma H, Chen ZM (2007) Anterior debridement and bone grafting of spinal tuberculosis with one-stage instrumentation anteriorly or posteriorly. Int Orthop 31: 859-863. [Crossref]

15. Moon M (2006) Development in the management of tuberculosis of the spine. Current Orthopaedics 20: 132-140.

16. Oguz E, Sehirlioglu A, Altinmakas M, Ozturk C, Komurcu M, et al. (2008) A new classification and guide for surgical treatment of spinal tuberculosis. Int Orthop 32: 127-33. [Crossref]

17. Christodoulou A, Givissis P, Karataglis D, D Symeonidis P, Pournaras J (2006) Treatment of tuberculous spondylitis with anterior stabilization and titanium cage. Clin Orthop Relat Res 444: 60-65. [Crossref]

Copyright: (C2020 Wang Bo. This is an open-access article distributed under the terms of the Creative Commons Attribution License, which permits unrestricted use, distribution, and reproduction in any medium, provided the original author and source are credited. 